\title{
Higher Prevalence of Periodontitis and Decayed, Missing and Filled Teeth in Patients with Psoriasis
}

\author{
Fabiana Cervo de Barros ${ }^{1,4}$ Janaina Nunes Sampaio \\ Sueli Carneiro ${ }^{3}$ Ricardo Guimarães Fischer ${ }^{1}$ \\ ${ }^{1}$ Department of Periodontology, Faculty of Dentistry, \\ Rio de Janeiro State University, Rio de Janeiro, Brazil \\ ${ }^{2}$ Division of Periodontology, School of Dentistry and Oral Health, \\ Griffith University, Queensland, Australia \\ ${ }^{3}$ Department of Dermatology, Faculty of Medical Sciences, \\ Rio de Janeiro State University, Rio de Janeiro, Brazil \\ ${ }^{4}$ Faculty of Dentistry, Arthur Sá Earp Neto University (FASE), \\ Petrópolis, Brazil
}

Eur J Dent:2020;14:366-370

\author{
Carlos Marcelo da Silva Figueredo ${ }^{1,2}$
}

\begin{abstract}
Keywords

- psoriasis

- periodontal disease

- decayed, missing and filled teeth index

- chronic diseases

Objective The aim of this study is to describe the prevalence and severity of periodontitis and decayed, missing and filled teeth (DMFT) index in patients with psoriasis. As a secondary aim, verify if periodontitis was a risk indicator for psoriasis.

Materials and Methods A total of 69 patients diagnosed with psoriasis (48.7 \pm 14.6 years) and 74 healthy controls ( $40.3 \pm 12.9$ years) participated in the study. Probing pocket depth, clinical attachment loss (CAL), bleeding on probing, plaque index, and DMFT index were measured in all subjects. Periodontitis was defined as the presence of at least three interproximal sites with CAL $\geq 3 \mathrm{~mm}$ in different teeth and severe periodontitis should involve at least two interproximal sites in different teeth with $C A L \geq 5 \mathrm{~mm}$.

Statistical Analysis The Mann-Whitney test was used to analyze the demographics and the clinical data. The significance level was $5 \%$. A multivariate logistic regression was conducted, and the odds ratio were calculated to express the risk to develop psoriasis.

Results Patients with psoriasis had significantly more sites with $\mathrm{CAL} \geq 3 \mathrm{~mm}(p<0.03)$ and $\mathrm{CAL} \geq 5 \mathrm{~mm}(p<0.0001)$, less sites with plaque $(p<0.0001)$, fewer teeth $(p<0.0001)$, and a high DMFT index $(p<0.02)$ as compared with controls. Severe periodontitis was significantly more frequent $(87.1 \% \times 58.1 \%)$ and was a risk indicator for psoriasis after adjusting for sex, age, race, and smoking habits (odds ratio: 3.7, 95\% confidence interval: $1.5-9.0, p<0.003$ ).

Conclusion Patients with psoriasis have higher prevalence of severe periodontitis and higher DMFT than control patients. Severe periodontitis may be a risk indicator for psoriasis.
\end{abstract}

\begin{abstract}
Address for correspondance Fabiana Cervo de Barros, DDS, MDSc, $\mathrm{PhD}$, Department of Periodontology, Faculty of Dentistry, Rio de Janeiro State University, Avenida Boulevard 28 de Setembro, 157, Vila Isabel, Rio de Janeiro 20551-030, RJ, Brazil (e-mail: fabianacervodebarros@gmail.com).
\end{abstract}

\section{Introduction}

Psoriasis is a chronic inflammatory disorder manifested in the skin and characterized by $\mathrm{T}$ cell-mediated hyperproliferation of keratinocytes. ${ }^{1}$ The prevalence is similar in male and female, affecting 1 to $3 \%$ of the population. ${ }^{2}$ It is considered a multifactorial disease, including infections, genetic, and behavior factors that may influence the dysfunction of the T cell expression and may begin the skin lesions. ${ }^{3}$ Periodontitis is an inflammatory disease involving the supporting tissues of the teeth, with connective tissue attachment and alveolar bone losses, that may lead to dental loss. Severe periodontitis affects 10 to $15 \%$ of the adult population. Both diseases have been associated with other chronic disease such as 
rheumatoid arthritis, diabetes mellitus, inflammatory bowel disease, arterial refractory hypertension, and cardiovascular diseases. ${ }^{4-8}$

In psoriasis patients, the oral lesions may occur before, during or after the dermatologic manifestation of the disease. ${ }^{9}$ Oral findings in these patients indicated high prevalence of fissured tongue, geographic tongue, and angular cheilitis. ${ }^{10}$ The dental and periodontal status may have an important influence in these patients. Sharma et $\mathrm{al}^{11}$ showed a high mean value of probing pocket depth, probing attachment loss and missing teeth in psoriasis group as compared with healthy group. Skudutyte-Rysstad et al $^{12}$ observed significantly higher prevalence of moderate and severe periodontitis in psoriasis individuals, as compared with healthy controls. Moreover, a significantly fewer teeth were also present in the psoriasis group. Preus et $\mathrm{al}^{13}$ described that patients with psoriasis have lower radiographic bone level and higher number of missing teeth than the control group. Santarelli et al $^{14}$ showed that psoriasis patients have an increased prevalence of gingivitis or periodontitis. On the other hand, Fadel et $\mathrm{al}^{15}$ related no differences in the profiles of caries and periodontal disease between individuals with or without psoriasis.

Both periodontitis and psoriasis share several risk factors, including smoking, obesity, and diabetes mellitus. ${ }^{16-18}$ Keller and Lin $^{19}$ and Nakib et $\mathrm{al}^{20}$ observed an increased risk for psoriasis among patients with chronic periodontitis. A systematic review and meta-analysis observed that individuals with periodontitis showed a significantly increased risk for developing psoriasis. ${ }^{21}$ In Brazil, no study has investigated the periodontal prevalence and risk of oral complications in such patients. Therefore, our aim was to describe the prevalence and severity of periodontitis and decayed, missing and filled teeth (DMFT) index in patients with psoriasis. As a secondary aim, the risk for patients with periodontal disease to develop psoriasis was analyzed.

\section{Materials and Methods}

Participated in this study 143 patients, 69 psoriasis patients were included in the case group ( $48.7 \pm 14.6$ years of age), while 74 healthy individuals were considered as control group ( $40.3 \pm 12.9$ years of age). Edentulous individuals, pregnant women, patients that suffered stroke or acute myocardial infarction were excluded. The patients should not have used antibiotics 6 months prior to the study. In the control group, individuals should not have received anti-inflammatory at least 3 months prior to the study. All subjects signed a term of consent. When the patient was younger than 18 years old, one of the parents or the guardians consented the participation in this work. The study was approved by the ethical committee of the Rio de Janeiro State University (protocol number: CEP/HUPE: 0026.0.228.000-11).
The patients were selected from the department of dermatology at the immunosuppressed and biological medicines ambulatory from Pedro Ernesto University Hospital of the Rio de Janeiro State University, and the diagnosis of psoriasis was done by a dermatologist. The systemic medication included methotrexate, cyclosporine, and/ or acitretin, diaminodiphenyl sulfone, fexofenadine or hydroxyzine hydrochloride. Thirteen patients with psoriasis were not taking any systemic medication at the moment of the study, but previously they had used immunosuppressed or biological medicine. Fifty-six patients received previously or were still under some topic treatment. The control group subjects did not show any clinical signs of ongoing systemic disease.

The individuals filled out a questionnaire with information about their age, gender, ethnicity, previous medical conditions (arterial hypertension, diabetes mellitus, chronic kidney disease, rheumatoid arthritis and inflammatory bowel disease, smoking habits, and oral history (including the last visit in dentist).

The clinical examination was performed in six sites of all present teeth (mesio-buccal, buccal, disto-buccal, disto-lingual, lingual, and mesio-lingual), except the third molars. The clinical data were assessed by the one examiner (FCB) using North Caroline periodontal probe (Hu-Friedy PC 15; Chicago, Illinois, United States) and included bleeding on probing (BOP), plaque index (PI), probing pocket depth, and CAL. BOP and PI were defined as present or absent, and the percentage of positive sites was calculated. The intraexaminer calibration has an agreement of $94 \%$ within the $\pm 1 \mathrm{~mm}$ for the measurements of PPD and CAL. Periodontitis was defined as the presence of, at least, three interproximal sites with CAL $\geq 3 \mathrm{~mm}$ in different teeth and severe periodontitis should involve, at least, two interproximal sites in different teeth with $\mathrm{CAL} \geq 5 \mathrm{~mm}$. The teeth were examined for decayed, missing and filled index (DMFT index) and the intraoral examination for assessment of mucosal lesions followed the World Health Organization. ${ }^{22}$ The oral lesions, such as fissured tongue and geographic tongue, were examined in the case group.

\section{Statistical Analysis}

Sample size calculation estimated a prevalence of periodontitis of 40 and $65 \%$ in the control and case groups, respectively. Considering a power of $80 \%$, the calculation included 70 patients on each group. The KolmogorovZmirnov Z normality test was applied to verify the distribution of the data. Normally distributed data were described as mean and standard deviation, while non-normally distributed data were described as median and interquartile range. The Mann-Whitney test was used to compare the demographics and the clinical data. A multivariate logistic regression was conducted, and the odds ratio were calculated to express the risk to develop psoriasis. The level of significance of $5 \%(p<0.05)$ was established. Statistical 
Package for Social Sciences 23 (SPSS, Inc.; Chicago, Illinois, United States) was used to calculate the data.

\section{Results}

The demographic data from case and control patients were reported in -Table 1. Patients in the case group were significantly older and presented a higher prevalence of arterial hypertension. There were 13 and 9 smokers in the case group and control group, respectively. There was no diabetic patient in both groups. Patients with psoriasis had $49 \%$ of fissured tongue and $16 \%$ of geographic tongue. Total $13 \%$ of the patients presented both lesions. Psoriasis patients had significantly fewer teeth $(p<0.0001)$, higher DMFT index $(p=0.02)$, higher number of sites with CAL $\geq 3 \mathrm{~mm}(p<0.03)$ and CAL $\geq 5 \mathrm{~mm}(p<0.0001)$, and lower number of sites with plaque $(p<0.0001)$ as compared with the control patients ( - Table 2 ). The prevalence of severe and generalized periodontitis was significantly higher in the case group.

Arterial hypertension (OR: 3.2, 95\% CI: 1.01-7.9, $p<0.009$ ) and severe periodontitis (OR: 3.7, 95\% CI: 1.5-9.0, $p<0.003$ ) were risk indicators for the presence of psoriasis after adjusting the model for sex, age, race, and smoking habits (-Table 3).

\section{Discussion}

In the present study, the extension and severity of periodontitis were significantly higher among psoriasis patients than the control group. This finding is in line with Sharma et $\mathrm{al}^{11}$ that showed significantly higher mean values of PPD and CAL among 33 psoriasis patients, as compared with 35 healthy subjects. The authors suggested that the periodontal status was associated with the severity of psoriasis. SkudutyteRysstad et $\mathrm{al}^{12}$ showed a significantly higher prevalence of

Table 1 Mean ( \pm standard deviation) for age and frequency of sex, race, smoking status and arterial hypertension in case $(n=69)$ and the control groups $(n=74)$

\begin{tabular}{|l|l|l|l|}
\hline \multirow{2}{*}{ Variable } & Case group & Control group & \multirow{2}{*}{-Value } \\
\cline { 2 - 3 } & $(\boldsymbol{n}=\mathbf{6 9 )}$ & $(\boldsymbol{n}=\mathbf{7 4 )}$ & \\
\hline Age (y) & $48.7( \pm 14.6)^{\mathrm{a}}$ & $40.3( \pm 12.9)$ & $<0.0001$ \\
\hline Sex female (\%) & 50 & 63.5 & 0.13 \\
\hline Race White (\%) & 37.1 & 38.2 & 0.9 \\
\hline Current smokers (\%) & 18.6 & 12.2 & 0.08 \\
\hline Arterial hypertension & $36.2^{\mathrm{a}}$ & 13.5 & 0.002 \\
\hline
\end{tabular}

aSignificantly higher than control.

Note: The Mann-Whitney test was used to analyze these data.

Table 2 Median (interquartile range) for the number of teeth; decayed, missing and filled tooth index; percentage of probing pocket depth, clinical attachment loss, sites with plaque and bleeding on probing in case $(n=69)$ and control $(n=74)$ groups

\begin{tabular}{|l|l|l|l|}
\hline \multirow{2}{*}{ Variable } & Case group & Control group & \multirow{2}{*}{-Value } \\
\cline { 2 - 3 } & $(\boldsymbol{n}=\mathbf{6 9})$ & $(\boldsymbol{n}=74)$ & \\
\hline Number of teeth & $21(13.5)^{\mathrm{a}}$ & $25(6.3)$ & $<0.0001$ \\
\hline Decayed & $1(2)$ & $2(4)$ & 0.04 \\
\hline Missing & $7(13.5)^{\mathrm{a}}$ & $3(6.3)$ & $<0.0001$ \\
\hline Filled & $4(7)$ & $5(6)$ & 0.43 \\
\hline DMFT index & $17(12)^{\mathrm{a}}$ & $12.5(11.3)$ & 0.02 \\
\hline \% of PPD $\geq 4 \mathrm{~mm}$ & $3.7(7.4)$ & $3.4(6.7)$ & 0.92 \\
\hline \% of PPD $\geq 6 \mathrm{~mm}$ & $0(1.8)$ & $0(1.3)$ & 0.72 \\
\hline \% of CAL $\geq 3 \mathrm{~mm}$ & $21.2(40.4)^{\mathrm{a}}$ & $10.9(26)$ & 0.03 \\
\hline \% of CAL $\geq 5 \mathrm{~mm}$ & $15.8(37.2)^{\mathrm{a}}$ & $2(7.8)$ & $<0.0001$ \\
\hline \% of sites with plaque & $27(28.7)^{\mathrm{b}}$ & $52.7(42.8)$ & $<0.0001$ \\
\hline \% of sites with BOP & $18.9(22)$ & $24.4(29.4)$ & 0.07 \\
\hline \% Severe periodontitis & $87.1^{\mathrm{a}}$ & 58.1 & $<0.0001$ \\
\hline \% Generalized periodontitis & $42.9^{\mathrm{a}}$ & 23 & 0.02 \\
\hline
\end{tabular}

Abbreviations: BOP, bleeding on probing; CAL, clinical attachment loss; DMFT, decayed, missing and filled tooth index; PPD, probing pocket depth.

aSignificantly higher than control group.

bSignificantly lower than control group.

Note: The Mann-Whitney test was used to evaluate these clinical data. 
Table 3 Odds ratio for the association of psoriasis and severe periodontitis

\begin{tabular}{|l|l|l|}
\hline & OR $(95 \% \mathrm{CI})$ & $p$-Value \\
\hline Smokers (yes) & $1.3(0.91-1.88)$ & 0.15 \\
\hline Arterial hypertension (yes) & $3.2(1.01-7.9)$ & 0.009 \\
\hline Sex (female) & $1.9(0.9-3.9)$ & 0.09 \\
\hline Age & $0.12(0.97-1.04)$ & 0.73 \\
\hline Severe periodontitis (yes) & $3.7(1.5-9.0)$ & 0.003 \\
\hline
\end{tabular}

Abbreviations: $\mathrm{Cl}$, confidence interval; OR, odds ratio.

moderate or severe periodontitis in a group of 50 psoriasis patients. Kathariya and Pradeep ${ }^{23}$ observed a higher prevalence of periodontitis in a psoriasis group, as compared with the control group. On the other hand, Fadel et $\mathrm{al}^{15}$ related no differences in the periodontal disease experience. The differences observed may be related to the methodology used. Sharma et al, ${ }^{11}$ Skudutyte-Rysstad et al, ${ }^{12}$ and our study examined four to six sites of all teeth, while Fadel et $\mathrm{al}^{15}$ examined only six teeth (community periodontal index of treatment needs), which usually underestimate the prevalence of periodontitis.

In our study, BOP was not significantly different between case and control groups. This finding is in line Fadel et al, ${ }^{15}$ although both groups of their study showed a suboptimal oral hygiene level. Although Skudutyte-Rysstad et $\mathrm{al}^{12}$ and Yarkac et $\mathrm{al}^{24}$ observed significantly higher mean values of BOP in the psoriasis group, Kathariya and Pradeep ${ }^{23}$ observed that PI and gingival index were significantly higher in the psoriasis group. In our study, $80 \%$ of the patients in the case group were using a systematic immunosuppressive medication. It may be suggested that the immunosuppressive and anti-inflammatory medications taken by the patients in the case group may have reduced the inflammatory tissue response. This fact was described in other studies dealing with chronic diseases. ${ }^{6,25}$ As Skudutyte-Rysstad et al, ${ }^{12}$ our study was not able to distinguish whether the observed differences could be attributed to the psoriasis or were a consequence of the medications used.

Severe periodontitis was a risk indicator for the development of psoriasis (odds ratio [OR]: 3.7, 95\% confidence interval $[\mathrm{CI}]: 1.5-9.0, p<0.003)$ in this population. Other studies showed similar results. Keller and Lin, ${ }^{19}$ in a retrospective cohort, showed an OR of 1.52 (95\% CI: 1.38-1.70; $p=0.05$ ) after adjusting. Lazaridou et $\mathrm{al}^{26}$ described an OR of 3.33 (95\% CI: $1.51-7.32 ; p=0.003$ ). Nakib et $\mathrm{al}^{20}$ observed an increased multivariate risk of psoriasis for those with moderate to severe periodontal bone loss relative risk of 1.49 (95\% CI: 1.08-2.05). Ungprasert et $\mathrm{a}^{21}$ in a meta-analysis observed that individuals with periodontitis had a significantly higher OR of 1.55 (95\% CI: 1.35-1.77) for developing psoriasis. Smoking is an important risk factor for periodontitis. However, in our study, smoking did not influence the results; as there was no significant difference of smokers among both groups and after the multivariate analysis, smoking was not considered a significant risk indicator.
The DMFT index was significantly higher among psoriasis patients as compared with the control group. Fadel et al ${ }^{15}$ described the same significant differences between both groups. There were no differences in the mean number of decayed teeth. The significantly higher number of missing teeth in the psoriasis group is in line with other studies ${ }^{11-13,23}$ and may reflect a worse dental maintenance. The age of the patients might have influenced a higher DMFT index. However, in our study, a multivariate analysis showed that age was not an important factor for higher DMFT index.

Limitations of this study include the selection of the case and the control groups. The case group was selected from patients attending a dermatological clinic at a hospital environment, while the control group was selected from the general population and it is less likely to have health problems. Also, the type and severity index of psoriasis were not informed; therefore, it was not possible to stablish a correlation with the psoriasis severity.

In conclusion, the present study showed that psoriasis patients had a higher prevalence of severe periodontitis and higher mean value of DMFT index, with more decayed and missing teeth, as compared with health controls. In addition, severe periodontitis may be a risk indicator to psoriasis.

\section{Conflict of Interest}

None declared.

\section{References}

1 Gudjonsson JE, Johnston A, Sigmundsdottir H, Valdimarsson H. Immunopathogenic mechanisms in psoriasis. Clin Exp Immunol 2004;135(1):1-8

2 Gelfand JM, Stern RS, Nijsten T, et al. The prevalence of psoriasis in African Americans: results from a population-based study. J Am Acad Dermatol 2005;52(1):23-26

3 Boyman O, Conrad C, Tonel G, Gilliet M, Nestle FO. The pathogenic role of tissue-resident immune cells in psoriasis. Trends Immunol 2007;28(2):51-57

4 Miranda LA, Fischer RG, Sztajnbok FR, Figueredo CM, Gustafsson A. Periodontal conditions in patients with juvenile idiopathic arthritis. J Clin Periodontol 2003;30(11):969-974

5 Mealey BL, Oates TW; American Academy of Periodontology. Diabetes mellitus and periodontal diseases. J Periodontol 2006;77(8):1289-1303

6 Brito F, de Barros FC, Zaltman C, et al. Prevalence of periodontitis and DMFT index in patients with Crohn's disease and ulcerative colitis. J Clin Periodontol 2008;35(6):555-560

7 Vidal F, Figueredo CM, Cordovil I, Fischer RG. Periodontal therapy reduces plasma levels of interleukin-6, C-reactive protein, and fibrinogen in patients with severe periodontitis and refractory arterial hypertension. J Periodontol 2009;80(5):786-791

8 Gottlieb AB, Dann F. Comorbidities in patients with psoriasis. Am J Med 2009;122(12):1150.e1-1150.e9

9 Younai FS, Phelan JA. Oral mucositis with features of psoriasis: report of a case and review of the literature. Oral Surg Oral Med Oral Pathol Oral Radiol Endod 1997;84(1):61-67

10 Lier GC, Mrowietz U, Wolfart M, Warnke PH, Wiltfang J, Springer IN. Psoriasis of the tongue. J Craniomaxillofac Surg 2009;37(1):51-53

11 Sharma A, Raman A, Pradeep AR. Association of chronic periodontitis and psoriasis: periodontal status with severity of psoriasis. Oral Dis 2015;21(3):314-319 
12 Skudutyte-Rysstad R, Slevolden EM, Hansen BF, Sandvik L, Preus HR. Association between moderate to severe psoriasis and periodontitis in a Scandinavian population. BMC Oral Health 2014;14:139-147

13 Preus HR, Khanifam P, Kolltveit K, Mørk C, Gjermo P. Periodontitis in psoriasis patients: a blinded, case-controlled study. Acta Odontol Scand 2010;68(3):165-170

14 Ganzetti G, Campanati A, Santarelli A, et al. Involvement of the oral cavity in psoriasis: results of a clinical study. $\mathrm{Br} J$ Dermatol 2015;172(1):282-285

15 Fadel HT, Flytström I, Calander AM, Bergbrant IM, Heijl L, Birkhed D. Profiles of dental caries and periodontal disease in individuals with or without psoriasis. J Periodontol 2013;84(4):477-485

16 Armstrong AW, Harskamp CT, Dhillon JS, Armstrong EJ. Psoriasis and smoking: a systematic review and meta-analysis. Br J Dermatol 2014;170(2):304-314

17 Christophers E. Comorbidities in psoriasis. Clin Dermatol 2007;25(6):529-534

18 Linden GJ, Lyons A, Scannapieco FA. Periodontal systemic associations: review of the evidence. J Periodontol 2013;84(4, Suppl):S8-S19

19 Keller JJ, Lin HC. The effects of chronic periodontitis and its treatment on the subsequent risk of psoriasis. Br J Dermatol 2012;167(6):1338-1344
20 Nakib S, Han J, Li T, Joshipura K, Qureshi AA. Periodontal disease and risk of psoriasis among nurses in the United States. Acta Odontol Scand 2013;71(6):1423-1429

21 Ungprasert P, Wijarnpreecha K, Wetter DA. Periodontitis and risk of psoriasis: a systematic review and meta-analysis. J Eur Acad Dermatol Venereol 2017;31(5):857-862

22 WHO, Oral health surveys: basic methodos. In: Path- finder Methodology. Geneva: World Health Organization; 1997 5-9

23 Kathariya R, Pradeep AR. Chronic plaque psoriasis and plaque-induced chronic periodontitis; is there any association: a cross-sectional study. J Periodontol Implant Dent 2011;3:13-20

24 Ucan Yarkac F, Ogrum A, Gokturk O. Effects of non-surgical periodontal therapy on inflammatory markers of psoriasis: A randomized controlled trial. J Clin Periodontol 2020;47(2):193-201

25 Ford PJ, Gamonal J, Seymour GJ. Immunological differences and similarities between chronic periodontitis and aggressive periodontitis. Periodontol 2000 2010;53:111-123

26 Lazaridou E, Tsikrikoni A, Fotiadou C, et al. Association of chronic plaque psoriasis and severe periodontitis: a hospital based case-control study. J Eur Acad Dermatol Venereol 2013;27(8):967-972 